\title{
Flocking-enhanced social contagion
}

\author{
Demian Levis, ${ }^{1,2, *}$ Albert Diaz-Guilera, ${ }^{1,2}$ Ignacio Pagonabarraga $\odot,{ }^{1,2,3}$ and Michele Starnini ${ }^{4, *, \dagger}$ \\ ${ }^{1}$ Departament de Fisica de la Materia Condensada, Universitat de Barcelona, Marti i Franques 1, 08028 Barcelona, Spain \\ ${ }^{2}$ Universitat de Barcelona Institute of Complex Systems (UBICS), Universitat de Barcelona, 08028 Barcelona, Spain \\ ${ }^{3}$ CECAM Centre Européen de Calcul Atomique et Moléculaire, Ecole Polytechnique Fédérale de Lausanne (EPFL), \\ Batochime, Avenue Forel 2, 1015 Lausanne, Switzerland \\ ${ }^{4}$ ISI Foundation, via Chisola 5, 10126 Torino, Italy
}

(Received 17 April 2020; accepted 15 July 2020; published 1 September 2020)

\begin{abstract}
Populations of mobile agents — animal groups, robot swarms, or crowds of people—self-organize into a large diversity of states as a result of information exchanges with their surroundings. While in many situations of interest the motion of the agents is driven by the transmission of information from neighboring peers, previous modeling efforts have overlooked the feedback between motion and information spreading. Here we show that such a feedback results in contagion enhanced by flocking. We introduce a reference model in which agents carry an internal state whose dynamics is governed by the susceptible-infected-susceptible (SIS) epidemic process, characterizing the spread of information in the population and affecting the way they move in space. This feedback triggers flocking, which is able to foster social contagion by reducing the epidemic threshold with respect to the limit in which agents interact globally. The velocity of the agents controls both the epidemic threshold and the emergence of complex spatial structures, or swarms. By bridging together soft active matter physics and modeling of social dynamics, we shed light upon a positive feedback mechanism driving the self-organization of mobile agents in complex systems.
\end{abstract}

DOI: 10.1103/PhysRevResearch.2.032056

Mobile agents-animals, people, or robots-interact through the exchange of information, which directly influences the way they move in space. These interactions trigger the emergence of a plethora of collective states, such as flocks of birds [1], robot swarms [2], or the coherent motion of fish schools avoiding a predator's attack [3]. All living entities use signaling inputs to get information about the environment. From their perception (visual, acoustic, quorum-sensing, etc.), individuals in a group might change the way they move according to the behavior of their surrounding peers, to capture the presence of potential threats or opportunities [4]. For animals, information could be related to the location of a food source or an approaching predator. In shoaling fish, for instance, social cues transmitted through the shoal have been suggested to enhance early predator detection [5]. In humans, the behavior of crowds in life-threatening situations can be determined by the spread of panic, transmitted locally among neighboring individuals [6,7]. At a smaller scale, for instance, cells regulate gene expression processing the input chemical signals received from their surroundings $[8,9]$.

The exchange of information in populations of mobile agents can be represented by the introduction of extra internal

\footnotetext{
${ }^{*}$ These authors contributed equally to this work.

${ }^{\dagger}$ Corresponding author: michele.starnini@gmail.com
}

Published by the American Physical Society under the terms of the Creative Commons Attribution 4.0 International license. Further distribution of this work must maintain attribution to the author(s) and the published article's title, journal citation, and DOI. degrees of freedom in models of active matter [10]. Active matter stands for systems composed of interacting units (active particles), that pump energy from their environment to self-propel, providing a simple modeling of living matter from a physics viewpoint $[11,12]$. Despite the relevance of the problems it raises, the study of active matter made of constituents exchanging information is still at its infancy. Artificial motile units featuring a feedback between their motion and some local information about the population have been engineered only in the last two years. These units might operate both at the microscale-colloids powered by external fields controlled by a feedback loop involving their positions [13-15] — and the macroscale_robot swarms mimicking pattern formation in biological systems [16]. Recently, very few works started considering simple models with a feedback between an agent's internal variable and the way they move in space. For instance, coupling the tendency to synchronize of globally coupled mobile phase oscillators with their spatial attraction produces novel self-sustained structures [17], while identifying the internal phase with the self-propulsion velocity of locally interacting active oscillators induces new synchronization phenomena, such as mutual flocking and chiral sorting $[18,19]$. Alternatively, the internal variable of the agents may represent opinions, whose dynamics depends on the spatial location of the agents which, in turn, is affected by their local social interactions [20], or a binary variable describing their state of motion (motile or not) [21].

In a completely different field, the dynamics of information spreading across a population has been modeled by paradigmatic epidemic processes [22], in which agents aware of the information (infected) transmit it to unaware (susceptible) 
neighboring individuals with certain probability. The information transmission is mediated by social interactions, naturally present in humans, but also observed in mammals $[23,24]$ and fish $[25,26]$. Information cascades in social animals may trigger the emergence of coordination and collective behavioral change [27], also indicated as behavioral or social contagion [28-30]. The collective motion of animal groups has been showed to be affected by the spreading of information within the group, encoding a preferred direction of motion of its leaders [31]. Within this framework, epidemic processes have been modeled also in systems of mobile agents, taking into account the fact that social interactions are not static but evolve in time. It has been shown that motion of the agents has a crucial impact on the spreading process [32-37]. However, in these setups, the coupling between the motility and the epidemic process has no feedback mechanism: the motion of the agents is not influenced by the epidemic spreading in any way.

However, while there is ample empirical evidence that information exchanges affect the way in which agents move in space $[27,31,38-40]$, a feedback mechanism has not been considered so far within the context of epidemic processes. Here, we propose a reference model that introduces a feedback between the agents' mobility and an internal degree of freedom, characterizing the information spreading across the population. Such feedback has a dramatic impact on the collective behavior of the population, both for its coordination in real space (e.g., collective motion, pattern formation) and in the abstract space of the internal states (e.g., consensus, epidemic outbreak). We consider pointlike self-propelled agents which carry an internal phase which evolves according to a susceptible-infected-susceptible (SIS) process [41]. Such phase represents the agent's tendency to move along a given direction, and thus directly influences its motion. We show that motility can enhance information spreading across a population and, conversely, information spreading induces a variety of cooperative states in real space, such as flocking and structure formation. Such rich and novel phenomenology crucially hinges on the feedback between the agents' mobility and the dynamics of their internal state.

We consider $N$ self-propelled agents moving in a $2 d$ volume $L \times L$ with periodic boundary conditions. At time $t$, agents (or active particles) are located at $\boldsymbol{r}_{i}(t)=\left[x_{i}(t), y_{i}(t)\right]$. They are self-propelled along their orientation $\boldsymbol{p}_{i}(t)=$ $\left[\cos \theta_{i}(t), \sin \theta_{i}(t)\right]$ with a constant velocity $v$ :

$$
\boldsymbol{r}_{i}(t+1)=\boldsymbol{r}_{i}(t)+v\left[\cos \theta_{i}(t), \sin \theta_{i}(t)\right],
$$

where $\theta_{i}$ is coupled to its internal phase, $\phi_{i}$, as

$$
\theta_{i}(t+1)=\theta_{i}(t)+H \sin \left[\phi_{i}(t)-\theta_{i}(t)\right] .
$$

The larger $H$, the faster $\theta_{i}$ adjust to $\phi_{i}$, for which evolution is driven by an underlying SIS process that we describe below. In the absence of coupling between the SIS process and the motion of the agents $(H=0)$, the agents move ballistically.

Each agent is endowed with an internal binary variable $s_{i}(t)=\{0,1\}$, representing its epidemic state, susceptible $\left(s_{i}=0\right)$ or infected $\left(s_{i}=1\right)$. At each time step, infected agents decay spontaneously to the susceptible state with probability $\mu$, while susceptible agents may become infected upon contact with infected neighbors with probability $\lambda$ [42]. Two

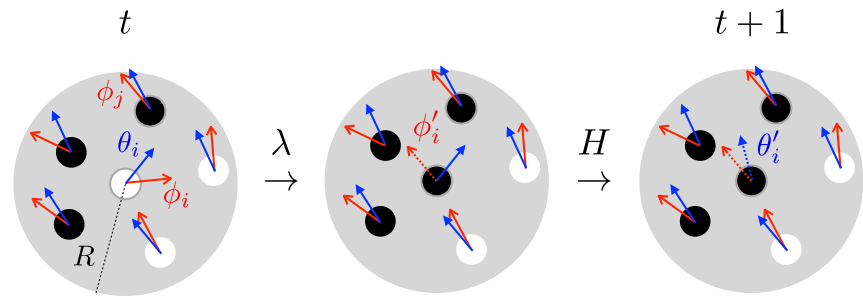

FIG. 1. Illustration of the interaction mechanism. Susceptible (infected) agents are represented in white (black). For each agent $i$, the internal phase $\phi_{i}$ (orientation $\theta_{i}$ ) is indicated by a red (blue) arrow. An initially susceptible agent $i$ gets infected with probability $\lambda$ by a neighboring agent $j$ and adopts its phase. Each agent aligns its orientation with its phase with a rate $H$.

agents $i$ and $j$ are neighbors if $\left|\boldsymbol{r}_{i}-\boldsymbol{r}_{j}\right|<R$. To couple such SIS process to the dynamics of $\phi$, let us now specify how the latter changes as a result of getting into a new epidemic state (or remaining in the same one). At a given time $t$, if an infected agent $i$ becomes susceptible, it picks a random phase $v \in[0 ; 2 \pi[$. This can be written as the following update of the internal variables of the system: $s_{i}(t)=1 \rightarrow s_{i}(t+1)=0$ and $\phi_{i}(t) \rightarrow \phi_{i}(t+1)=v$, which occurs with probability $\mu$. An infected agent that remains infected does not change its internal state at all, meaning $s_{i}(t+1)=s_{i}(t)=1$ and $\phi_{i}(t+$ $1)=\phi_{i}(t)$, with probability $1-\mu$. Then if an agent $i$ gets infected by a neighbor $j \in \partial_{i}$, it adopts the internal phase of $j: s_{i}(t)=0 \rightarrow s_{i}(t+1)=1$ and $\phi(t) \rightarrow \phi_{i}(t+1)=\phi_{j}(t)$, with probability $\lambda$. Finally, the phase of susceptible agents performs Brownian motion with diffusivity $D_{0}$ as long as they do not get infected. All in all, the evolution of the internal variables $\left\{\phi_{i}, s_{i}\right\}$ can be formally recast in the following compact form:

$$
\left\{\phi_{i}, s_{i}\right\}(t+1)= \begin{cases}\{v, 0\}, & \mu s_{i}(t) \\ \left\{\phi_{i}(t), 1\right\}, & (1-\mu) s_{i}(t) \\ \left\{\phi_{j \in \partial_{i}}(t), 1\right\}, & \lambda\left[1-s_{i}(t)\right] \\ \left\{\phi_{i}(t)+\delta \phi, 0\right\}, & (1-\lambda)\left[1-s_{i}(t)\right],\end{cases}
$$

where $\delta \phi=\sqrt{2 D_{0}} \mathcal{N}(0,1)$ is a Gaussian white noise term and the rightmost column indicates the transition probabilities.

The crucial new feature of the present model is the coupling between an internal state, governed by the SIS process, and motility. To illustrate the interaction mechanism, depicted in Fig. 1, let us consider agents as mimicking fish. At time $t$, an agent $i$, located in $\boldsymbol{r}_{i}$, is infected (e.g., aware of the presence of food) and has its orientation $\theta_{i}$ directed along its internal phase $\phi_{i}$, pointing toward its goal (the food source). At time $t+1$, with probability $\lambda$, agent $i$ transmits this information to a neighbor $j, \phi_{j}(t+1)=\phi_{i}(t)$, which in turn adjusts its orientation $\theta_{j}$. At the same time, agent $i$ may forget the information with small probability $\mu$, its internal phase decaying to a random state. This change can be triggered by environmental factors, and encodes the fact that, after some time, the information might not be relevant anymore. Depending on the effective infection rate $\lambda / \mu$, the information may spread further, or die out. The feedback mechanism is crucial here: the more agents share the same phase $\phi$, the more move along the same direction and the more effective 

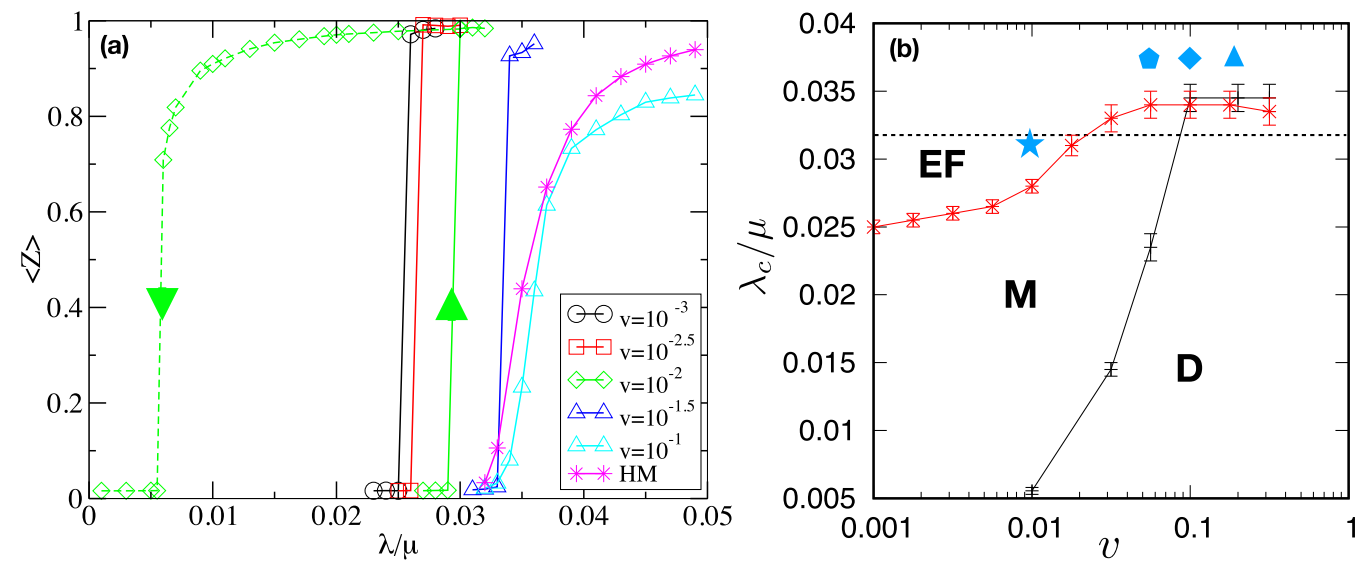

FIG. 2. (a) Polar order parameter $\langle Z\rangle$ as a function of the effective infection ratio $\lambda / \mu$ for several values of $v$, as obtained from a high- $\lambda$ (continuous line) and a low- $\lambda$ quench (dashed line). The homogeneous mixing (HM) curve has been obtained by changing randomly the position of all agents at each time step. (b) Epidemic thresholds $\lambda_{c}^{-}$(red) and $\lambda_{c}^{+}$(black) corresponding to the onset of flocking obtained from a high- $\lambda$ and a low- $\lambda$ quench, respectively. One can distinguish the endemic flocking (EF), metastable (M), and disordered (D) regions. The four symbols correspond to the four snapshots shown in Fig. 3.

the information spreads. Our model thus mimics the behavior of groups of animals [27], or crowds of people [43], and is responsible for the propagation of information concerning an external stimuli, for instance the presence of a threat [3] or the location of an exit in panic situations [7]. Agents receiving the stimuli first, for example the animals close to the food source, adjust their direction of motion towards it. Neighboring agents then adjust their direction of motion accordingly and the information about the food location spreads like an epidemic process.

A configuration of the system is given by $\Gamma(t)=$ $\{\boldsymbol{r}(t), \theta(t), \phi(t), s(t)\}_{i}$, while its control parameters are the self-propulsion velocity $v$; the average density $\rho_{0}=N / L^{2}$; the rates $\lambda, \mu$, and $H$; the diffusivity $D_{0}$; and the interaction range $R$. We fix $\mu=0.1, \rho_{0}=10, D_{0}=5 \times 10^{-4}$, and $R=$ 1 , while systematically exploring the behavior of the model by varying $\lambda, H$, and $v$ for systems of $N=10^{3}$ up to $3 \times 10^{4}$ agents (see [44] for details).

We explore the steady states of the model as a function of the polarization $Z(t) \equiv N^{-1}\left|\sum_{i} e^{i \theta_{i}(t)}\right|$ and the prevalence $n(t) \equiv N^{-1} \sum_{i} s_{i}(t)$. As $\lambda$ increases, the system undergoes a phase transition from a disordered state, where most agents are susceptible and move randomly (see [44] for the dynamics of the system in the diluite limit), to an endemic flocking ordered state, characterized by a macroscopic number of agents moving coherently along a preferred direction and a finite fraction of infected agents, i.e., $Z>0$ and $n>0$.

Therefore, the feedback between the epidemic process and the motility of the agents drives flocking, as extensively studied in Vicsek-like models [45,46]. The latter (and other models of flocking $[47,48]$ ), consider agents which align their velocity with their neighbors. Here, it is the feedback between the SIS process and motility which eventually leads to collective motion, once a fraction of the system is "infected," rather than an explicit velocity alignment, which is absent in our model.

Figure 2(a) shows the polarization $\langle Z\rangle$ as a function of $\lambda / \mu$, for different values of $v$ at fixed $H=0.01$ (where $\langle *\rangle$ denotes an average over steady states). Such data was obtained from two different protocols: (i) starting from a disordered configuration and letting the system relax towards its steady state, i.e., a high- $\lambda$ quench, and (ii) starting from an ordered state, i.e., a low- $\lambda$ quench. Starting from a disordered state and doing a high- $\lambda$ quench, the order parameter jumps from $\langle Z\rangle \approx$ 0 to $\langle Z\rangle \approx 1$ at a given value of $\lambda=\lambda_{c}^{+}$, which sets the limit of stability of the disordered state. Conversely, when letting the system relax after a low- $\lambda$ quench, the order parameter jumps from $\langle Z\rangle \gtrsim 0.7$ to $\langle Z\rangle \approx 0$ at $\lambda=\lambda_{c}^{-}$, indicating the limit of stability of the ordered state. Typically, $\lambda_{c}^{-}<\lambda_{c}^{+}$, a behavior is consistent with a discontinuous, or first-order, phase transition: hysteresis and abrupt changes of the order parameter around the transition. Following the first-order transition picture, the two instability lines $\lambda_{c}^{ \pm}$, or spinodals, define a metastable region in the $\lambda-v$ plane, shown in Fig. 2(b).

Moreover, as shown in Fig. 2(b), the metastability region between $\lambda_{c}^{-}$and $\lambda_{c}^{+}$shrinks as $v$ increases, and vanishes for $v \gtrsim 0.1$, consistently with a continuous transition. (Analog behavior is found for the prevalence $\langle n\rangle$; see [44]). Indeed, at large $v$ the system approaches the limit of homogeneous mixing [36], in which the positions of the agents are randomly updated independently of the SIS dynamics, thus breaking the feedback between motility and the SIS process. The epidemic threshold in this limit is $\lambda_{c}^{H M} / \mu=\left(\pi R^{2} \rho\right)^{-1}(\approx 0.0318$ in our case). Our model at finite $v$ shows a much lower threshold over a broad range of parameters, demonstrating that the feedback between motility and contagion triggers flocking, which is able to enhance social contagion. For large velocities, instead, the epidemic threshold approaches $\lambda_{c}^{H M}$ (the threshold reported is slightly larger than $\lambda_{c}^{H M}$ because of the numerical accuracy of its identification).

Note that previous models in which the agents mobility is decoupled from the epidemic dynamics have reported larger epidemic thresholds with respect to the homogeneous mixing case [36], or nonmonotonous behavior of the threshold with respect to the agents' velocity [37]. In the absence of feedback, the agents have to move fast enough to allow for an endemic state to emerge. Thus, the epidemic threshold increases if the velocity of the agents is reduced [34]. In 


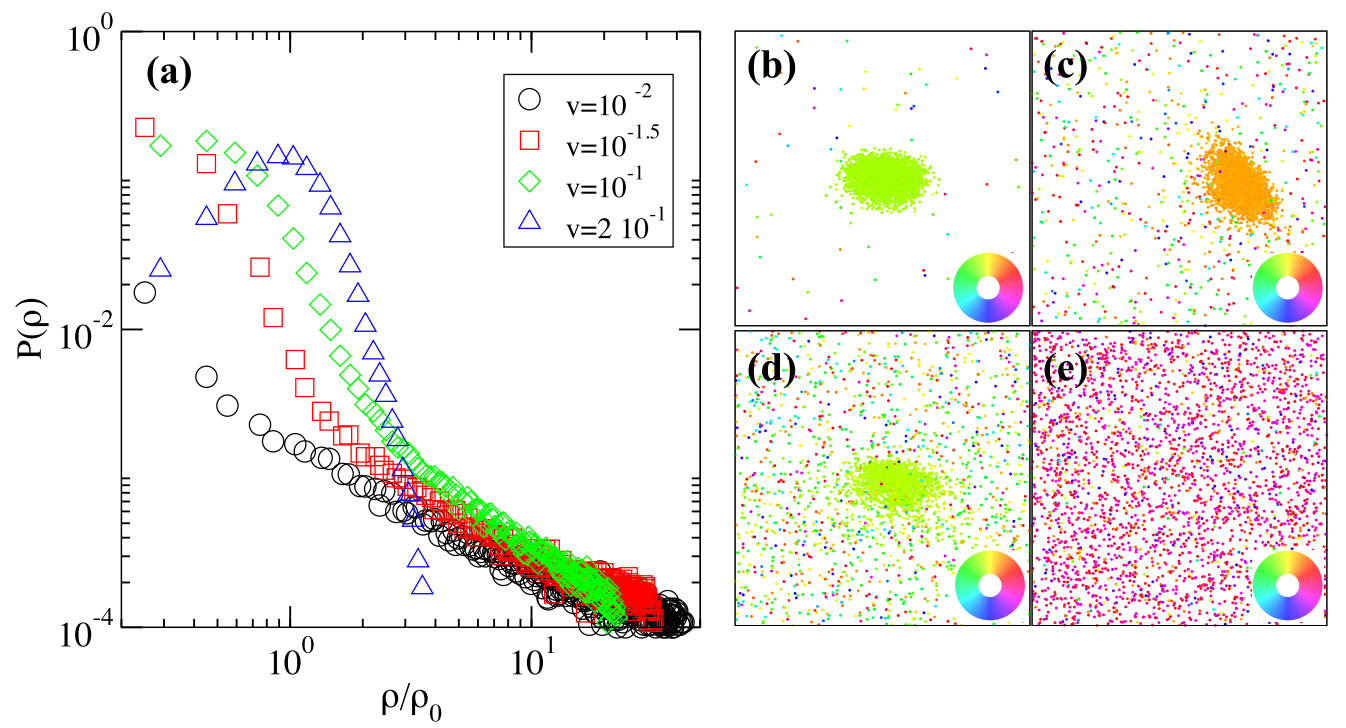

FIG. 3. Density distribution $P(\rho)$ for a system with different $v$ but fixed $\left(\lambda-\lambda_{c}^{+}\right) / \lambda_{c}^{+}=0.10$ and $H=0.01$ (a), together with representative snapshots at $v=0.01$ (b), 0.0562 (c), 0.1 (d), and 0.2 (e). These snapshots correspond to the four points marked by symbols in the $\lambda-v$ phase space, in Fig. 2(b). Each agent is represented by a color corresponding to its orientation $\theta_{i}$. The location of the snapshots in the $(\lambda, v)$ plane is indicated in Fig. 2(b).

contrast, in the presence of feedback as in our case, decreasing the velocity reduces the epidemic threshold. Complex, nonmonotonic behavior of the synchronization threshold as a function of the velocity has also been found in systems of mobile robots $[49,50]$. It is worth noticing that the static limit of our model is singular and our results cannot be extrapolated to $v \rightarrow 0$ (see [51] for a precise study of this limit for the Vicsek model).

In the low velocity regime $(v \lesssim 0.1)$, neighboring agents interact with each other for long periods of time, favoring contagion at short distances and leading to coordinated motion: we thus call it the flocking-enhanced contagion regime. Flocking increases the interaction time as compared to agents moving independently of their epidemic state, thus fostering contagion. This positive feedback loop is at the origin of the reduction of the epidemic threshold and the enhancement of flocking, due to the presence of dense swarms (clusters of aligned particles) in a gaseous (incoherent) background. In Fig. 3, we show typical snapshots obtained for different velocities at a fixed distance to the transition $\left(\lambda-\lambda_{c}^{+}\right) / \lambda_{c}^{+}=0.10$, together with the corresponding local density distributions $P(\rho)$. As shown in Fig. 3, the slower the particles are, the denser the swarms, the more dilute is the surrounding medium and the more efficient is the local contagion. This is evidenced by the long tails of $P(\rho)$, which are pushed to higher densities as $v$ decreases, while the peak of $P(\rho)$ at small $\rho$ approaches zero and becomes sharper. In the limit of large velocities, the spatial structure of the system is lost, the motion of the agents and the SIS process decouple, and thus local structures are no longer possible. As illustrated in Fig. 3(e), for $v=0.2, P(\rho)$ is Gaussian and the system does not feature any swarm but it is in a homogeneous flocking state. Interestingly, $P(\rho)$ shows a crossover from Gaussian behavior, to a power-law-like one, which develops as soon as swarms appear. These structures exhibit large density fluctuations (they are constantly reshaping) and are responsible for the algebraiclike tails of $P(\rho)$ in the flocking-enhanced contagion regime.

The observation of swarms, hysteresis, and order parameter jumps in our model is reminiscent of the Vicsek model phenomenology [48], although in a different parameter space and driven by an SIS process. For systems with periodic boundary conditions, the discontinuous character of the flocking transition in the Vicsek model is understood as arising from the microphase separation of the system into band-shaped swarms [52-54]. Structure formation in our model is in contrast with this scenario. The absence of hysteresis and the smoothing of the polarization curves for large velocities suggests the transition changes from discontinuous to continuous for $v \gtrsim$ 0.1 . Indeed, at low velocities, the system displays swarms, with a shape that differs from bands (typical in Vicseklike models), while at larger velocities the ordered phase is homogeneous. Such change in morphology shows that the ordering mechanism in the fast and slow velocity regimes is qualitatively different. It is worth noting that, in the context of Vicsek-like models, the amplitude of the self-propulsion velocity $v$ is not expected to shift the onset of flocking, or the nature of the transition (as long as $v>0$ ), but only the growth rate of the associated long-wavelength instability [55]. However, in our model, the value of $v$ affects the location of the transition and, moreover, its nature (and thus the structure of the ordered state, as shown in Fig. 3).

Due to the absence of excluded volume effects in our model, the local density of the swarms is not bounded. Obviously, steric interactions are likely to play an important role in dense situations such as robot swarms like in [2,16], or crowds of people [6,7]. The pointlike description taken here is suitable for describing dilute systems, such as the Vicsek model and its numerous extensions [56]. It is worth stressing that, here, our aim is to introduce a feedback between the agents mobility and information spreading in the simplest possible way, in 


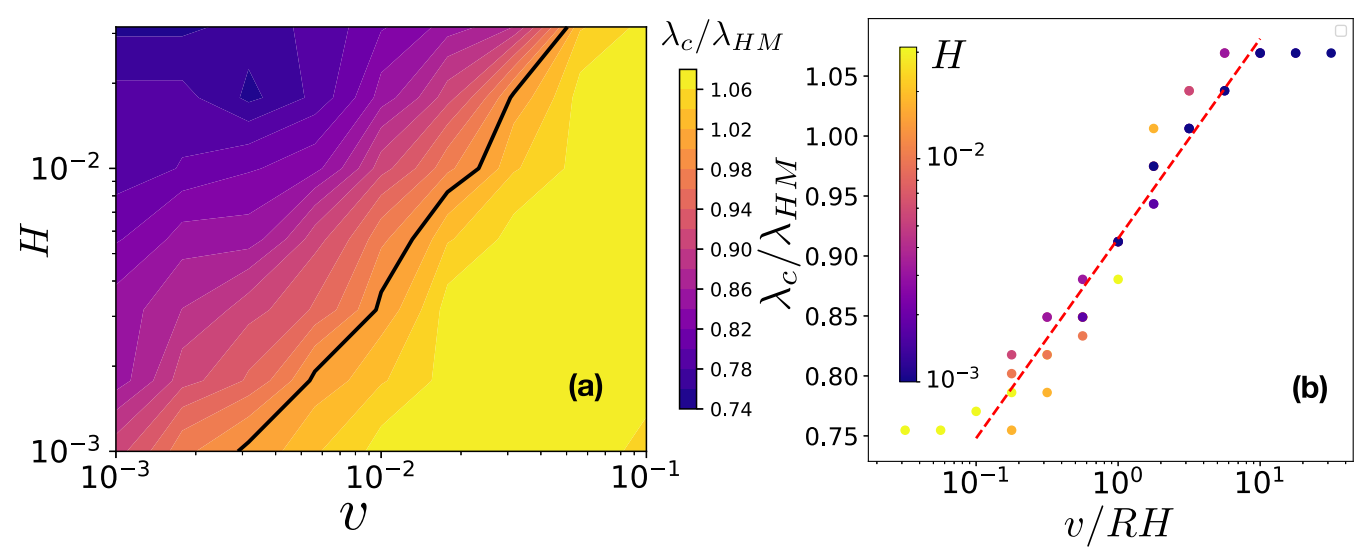

FIG. 4. (a) Contour lines associated to the limit of stability of the disordered state $\lambda_{c}^{+}$in the $(v-H)$ plane (in log-log scale). The homogeneous mixing limit $\lambda_{c}^{H M}$ is plotted as a continuous line. (b) Rescaled epidemic threshold $\lambda_{c} / \lambda_{H M}$ as a function of the scaling parameter $v / R H$, according to Eq. (4). A logarithmic scaling function is plotted as a dashed line.

order to be able to disentangle the role of such feedback from more complex mechanisms rooted on many-particle collisions. Indeed, collisions introduce a complex coupling between density and orientation fluctuations at short length scales which considerably affects the structure of the system, as the literature on systems of aligning self-propelled particles with excluded volume interactions teaches us [57-59].

The epidemic threshold also depends on the strength of the phase-orientation coupling: the larger $H$, the faster the agents adjust $\theta_{i}$ with their phase $\phi_{i}$, promoting the contagion. The impact of both, the phase-orientation coupling $H$ and $v$, on the emergence of flocking, is summarized in Fig. 4(a).

The curve corresponding to the homogeneous mixing threshold $\lambda_{c}^{H M}$ is highlighted as a solid black line. The critical threshold $\lambda_{c}^{+}$decreases as $v$ decreases and $H$ increases, with a minimum reached at large $H$ and low $v$. Interestingly, $H$ and $v$ play an opposite role as for the onset of flocking. This can be understood with the following simple argument. The typical time during which an infected agent (which moves ballistically, its self-propulsion direction being aligned with its internal phase) interacts with its neighborhood is $t_{I} \propto R / v$. For the onset of flocking to emerge, the interaction time has to be longer than the relaxation time $H^{-1}$ of the orientations. Once the orientation of newly infected agents has been locked, they move together and are able to collect more susceptible agents into the growing flock. As agents move faster, their interaction time is reduced, but this can be counterbalanced by an increase of $H$. The comparison between these timescales suggests that, in the flocking-enhanced contagion regime, the epidemic threshold exhibits the following scaling:

$$
\lambda_{c}=f\left[\frac{v}{R H}\right] .
$$

To put this hypothesis to the test, in Fig. 4(b) we plot $\lambda_{c} / \lambda_{H M}$ as a function of $v / R H$, showing that Eq. (4) holds at least for two orders of magnitude. Note that for large values of $v / R H \gtrsim 10$ we reach the homogeneous mixing limit (with $\lambda_{c}$ slightly larger than $\lambda_{H M}$ ), while for $v / R H \lesssim 10^{-1}$ we approach the static limit, which is singular. Interestingly, the scaling function $f(v / R H)$ seems to be compatible with a logarithm [dashed line in Fig. 4(b)], indicating a weak growth of the epidemic threshold with $v$. Note, however, that in the flocking-enhanced regime the epidemic threshold is reduced up to $25 \%$ with respect to the homogeneous mixing limit.

To sum up, we showed how a feedback mechanism between motility and information spreading, expected to be at play in many empirical populations of mobile agents, can trigger flocking and enhance social contagion. To illustrate this framework, we proposed a reference model built on the basis of paradigmatic models of active matter and epidemic spreading, providing a thorough characterization of its largescale behavior. The agent's velocity $v$ crucially controls the epidemic threshold and the nature of the (endemic flocking) phase transition: as $v$ increases, the critical point increases and its nature changes from discontinuous to continuous. The ordering mechanism for fast and slow agents (as compared with the intrinsic contagion timescale) is qualitatively different and allows one to control the formation of rich spatiotemporal patterns, or swarms. Our results provide a consistent picture about the role played by the main parameters of the model, the velocity $v$ and the strength of the phaseorientation coupling $H$, which can be rescaled in a single parameter.

The present work unveils the effects of a positive feedback between self-propulsion and information spreading in populations of mobile agents. Such feedback is responsible for the enhancement of information spreading and the emergence of rich spatiotemporal patterns. As such, this study bridges together soft active matter physics and the modeling of social dynamics, pushing forward the knowledge boundaries of both fields. The proposed framework shed light upon a feedback mechanism driving the self-organization of mobile agents in complex systems, such as animal groups, crowds of people, or robot swarms. In future work, we aim at comparing the model's behavior with empirical data collected in real systems of mobile agents subjected to an external perturbation, such as animal groups facing a predator attack $[40,60]$. Overall, our results shed light upon the effects of the interplay between information spreading and motility, and may thus constitute a guideline to design strategies for populations of artificial mobile agents (such as centimeter-sized robots $[2,16]$ ) with targeted functionalities. 
D.L. acknowledges MCIU/AEI/FEDER for financial support under Grant Agreement No. RTI2018-099032-J-I00. A.D.-G. acknowledges MINECO for financial support under Project No. FIS2015-71582-C2-2-P. I.P. acknowledges
MICINN, DURSI and SNSF for financial support under Projects No. PGC2018-098373-B-I00, No. 2017SGR-884, and No. 200021-175719, respectively.
[1] D. J. T. Sumpter, Collective Animal Behavior (Princeton University Press, Princeton, NJ, 2010).

[2] M. Rubenstein, A. Cornejo, and R. Nagpal, Science 345, 795 (2014).

[3] C. C. Ioannou, V. Guttal, and I. D. Couzin, Science 337, 1212 (2012).

[4] D. J. Pearce, A. M. Miller, G. Rowlands, and M. S. Turner, Proc. Natl. Acad. Sci. USA 111, 10422 (2014).

[5] A. Chicoli and D. A. Paley, Chaos 26, 116311 (2016).

[6] D. Helbing, I. Farkas, and T. Vicsek, Nature (London) 407, 487 (2000).

[7] D. J. Low, Nature (London) 407, 465 (2000).

[8] G. Tkačik, C. G. Callan, and W. Bialek, Proc. Natl. Acad. Sci. USA 105, 12265 (2008).

[9] G. Micali and R. G. Endres, Curr. Opin. Microbiol. 30, 8 (2016).

[10] M. R. Shaebani, A. Wysocki, R. G. Winkler, G. Gompper, and H. Rieger, Nat. Rev. Phys. 2, 181 (2020).

[11] S. Ramaswamy, Annu. Rev. Condens. Matter Phys. 1, 323 (2010).

[12] M. C. Marchetti, J. F. Joanny, S. Ramaswamy, T. B. Liverpool, J. Prost, M. Rao, and R. A. Simha, Rev. Mod. Phys. 85, 1143 (2013).

[13] U. Khadka, V. Holubec, H. Yang, and F. Cichos, Nat. Commun. 9, 3864 (2018).

[14] T. Bäuerle, A. Fischer, T. Speck, and C. Bechinger, Nat. Commun. 9, 3232 (2018).

[15] F. A. Lavergne, H. Wendehenne, T. Bäuerle, and C. Bechinger, Science 364, 70 (2019).

[16] I. Slavkov, D. Carrillo-Zapata, N. Carranza, X. Diego, F. Jansson, J. Kaandorp, S. Hauert, and J. Sharpe, Sci. Robotics 3, eaau9178 (2018).

[17] K. P. O'Keeffe, H. Hong, and S. H. Strogatz, Nat. Commun. 8, 1504 (2017).

[18] D. Levis, I. Pagonabarraga, and B. Liebchen, Phys. Rev. Res. 1, 023026 (2019).

[19] D. Levis and B. Liebchen, Phys. Rev. E 100, 012406 (2019).

[20] M. Starnini, M. Frasca, and A. Baronchelli, Sci. Rep. 6, 31834 (2016).

[21] M. Paoluzzi, M. Leoni, and M. C. Marchetti, Phys. Rev. E 98, 052603 (2018).

[22] R. Pastor-Satorras, C. Castellano, P. Van Mieghem, and A. Vespignani, Rev. Mod. Phys. 87, 925 (2015).

[23] D. Lusseau and M. E. J. Newman, Proc. R. Soc. London. Ser. B 271, 477 (2004)

[24] J. C. Flack, D. C. Krakauer, and F. B. M. de Waal, Proc. R. Soc. London, Ser. B 272, 1091 (2005).

[25] D. P. Croft, R. James, and J. Krause, Exploring Animal Social Networks (Princeton University Press, Princeton, UK, 2008).

[26] D. P. Croft, R. James, A. J. W. Ward, M. S. Botham, D. Mawdsley, and J. Krause, Oecologia 143, 211 (2005).

[27] S. B. Rosenthal, C. R. Twomey, A. T. Hartnett, H. S. Wu, and I. D. Couzin, Proc. Natl. Acad. Sci. USA 112, 4690 (2015).
[28] P. S. Dodds and D. J. Watts, Phys. Rev. Lett. 92, 218701 (2004).

[29] A. L. Hill, D. G. Rand, M. A. Nowak, and N. A. Christakis, PLoS Comput. Biol. 6, e1000968 (2010).

[30] C. Castellano, S. Fortunato, and V. Loreto, Rev. Mod. Phys. 81, 591 (2009).

[31] I. D. Couzin, J. Krause, N. R. Franks, and S. A. Levin, Nature (London) 433, 513 (2005).

[32] M. González and H. Herrmann, Phys. A (Amsterdam, Neth.) 340, 741 (2004).

[33] M. J. Keeling and P. Rohani, Modeling Infectious Diseases in Humans and Animals (Princeton University Press, Princeton, NJ, 2011).

[34] F. Peruani and G. J. Sibona, Phys. Rev. Lett. 100, 168103 (2008).

[35] M. Frasca, A. Buscarino, A. Rizzo, L. Fortuna, and S. Boccaletti, Phys. Rev. E 74, 036110 (2006).

[36] A. Buscarino, L. Fortuna, M. Frasca, and V. Latora, Europhys. Lett. 82, 38002 (2008).

[37] J. P. Rodríguez, F. Ghanbarnejad, and V. M. Eguíluz, Sci. Rep. 9, 6463 (2019).

[38] I. D. Couzin, C. C. Ioannou, G. Demirel, T. Gross, C. J. Torney, A. Hartnett, L. Conradt, S. A. Levin, and N. E. Leonard, Science 334, 1578 (2011).

[39] N. O. Handegard, K. M. Boswell, C. C. Ioannou, S. P. Leblanc, D. B. Tjøstheim, and I. D. Couzin, Curr. Biol. 22, 1213 (2012).

[40] A. Procaccini, A. Orlandi, A. Cavagna, I. Giardina, F. Zoratto, D. Santucci, F. Chiarotti, C. K. Hemelrijk, E. Alleva, G. Parisi et al., Anim. Behav. 82, 759 (2011).

[41] W. O. Kermack and A. G. McKendrick, Proc. R. Soc. London, Ser. A 115, 700 (1927).

[42] If a susceptible agent is surrounded by more than one infectious neighbor, each connection hosts statistically independent infection processes.

[43] L. B. Rosenberg, in 2015 Swarm/Human Blended Intelligence Workshop (SHBI) (IEEE, New York, 2015), pp. 1-7.

[44] See Supplemental Material at http://link.aps.org/supplemental/ 10.1103/PhysRevResearch.2.032056 for supplementary information about numerical simulations and analytical limits.

[45] T. Vicsek, A. Czirók, E. Ben-Jacob, I. Cohen, and O. Shochet, Phys. Rev. Lett. 75, 1226 (1995).

[46] J. Toner and Y. Tu, Phys. Rev. Lett. 75, 4326 (1995).

[47] T. Vicsek and A. Zafeiris, Phys. Rep. 517, 71 (2012).

[48] F. Ginelli, Eur. Phys. J.: Spec. Top. 225, 2099 (2016).

[49] F. Perez-Diaz, R. Zillmer, and R. Groß, Phys. Rev. Appl. 7, 054002 (2017).

[50] F. Perez-Diaz, R. Zillmer, and R. Groß, in Proceedings of the 2015 International Conference on Autonomous Agents and Multiagent Systems, AAMAS '15 (International Foundation for Autonomous Agents and Multiagent Systems, Richland, SC, 2015), pp. 279-286.

[51] G. Baglietto and E. V. Albano, Comput. Phys. Commun. 180, 527 (2009). 
[52] G. Grégoire and H. Chaté, Phys. Rev. Lett. 92, 025702 (2004).

[53] H. Chaté, F. Ginelli, G. Grégoire, and F. Raynaud, Phys. Rev. E 77, 046113 (2008).

[54] A. P. Solon, H. Chaté, and J. Tailleur, Phys. Rev. Lett. 114, 068101 (2015).

[55] E. Bertin, M. Droz, and G. Grégoire, J. Phys. A 42, 445001 (2009).

[56] H. Chaté, Ann. Rev. Condens. Matter Phys. 11, 189 (2020).
[57] F. Peruani, T. Klauss, A. Deutsch, and A. Voss-Boehme, Phys. Rev. Lett. 106, 128101 (2011).

[58] F. D. C. Farrell, M. C. Marchetti, D. Marenduzzo, and J. Tailleur, Phys. Rev. Lett. 108, 248101 (2012).

[59] A. Martín-Gómez, D. Levis, A. Díaz-Guilera, and I. Pagonabarraga, Soft Matter 14, 2610 (2018).

[60] A. Cavagna, A. Cimarelli, I. Giardina, G. Parisi, R. Santagati, F. Stefanini, and M. Viale, Proc. Natl. Acad. Sci. USA 107, 11865 (2010). 